\title{
The Antioxidant in Blueberry Fruits - A Combat to Overcome Aging and Neurodegenerative Diseases
}

\author{
Shimon Shatzmiller* \\ Department of Chemical Sciences, Ariel University, Ariel, Israel \\ *Corresponding Author: Shimon Shatzmiller, Department of Chemical Sciences, Ariel University, Ariel, Israel.
}

Received: October 22, 2019; Published: November 13, 2019

DOI: 10.31080/ASNE.2019.02.0125

\begin{abstract}
Phenols type antioxidants are an essential part of our daily diet. Their function as antioxidants protects our body from any unwanted diseases like cancer or neurodegenerative diseases. Many fruits and vegetables are the naturals source of the essential compounds. In this review, we focus on a few sorts od phenolic antioxidants and mainly appreciate the contribution of forest growing berries like blueberries for the maintenance of our brain health.
\end{abstract}

Keywords: Antioxidant Blood-Brain Barrier Crossing Resveratrol

\section{Introduction}

Research on the effects of dietary polyphenols on human health has considerably developed the 10 years. It strongly supports the role Polyphenols [1] in the prevention of degenerative diseases, in particular Cardiovascular disease and neurodegeneration.

There is nowadays evidence that blueberries have powerful fighting features. The tiny fruit (Blueberries aren't actually blue, but deep purple) has recently caught the attention of researchers, and is now attributed to health benefits such as high antioxidant activity [2], aging properties [3], ability to improve vision [4] and brain function [5] Lipid-lowering [6].

Oxidative stress and reactive oxygen speciesa

Although oxygen is the main requirement of all aerobic life forms on Earth, its intermediates, when found at higher than average concentrations in biological systems, can potentially damage cells that could eventually cause cell death. Any molecular species that contains electrons or is not polluted in its most visible shell and can exist independently is called free radicals [7]. Reactive oxygen species (ROS) may be free radicals, and are intermediates of oxygen created when oxygen is activated by physical or chemical means [8]. When the thoracic oxygen state is physically activated by the energy transfer, the oxygen molecule earns enough energy to change its spin and turn to ROS, that is, singlet oxygen. Chemical activation occurs when the oxygen molecule is reduced stepby-step and intermediate products are activated and superoxide radicals, which is the first intermediate, hydrogen peroxide, which itself is not very reactive but is radically reduced to very hydroxyl reactive [9]. The production of ROS during breathing is inevitable since the mitochondria electron transport chain involves the direct reduction of oxygen by the free energy of the electrons [10]. Of total $\mathrm{O}_{2}$ consumption by plant tissue, an estimated $1 \%$ contributes to the ROS generation [11]. In mitochondria, I and III complexes are responsible for the production of ROS, especially the IO which is disposed of to a less toxic but more widespread form of ROS, namely hydrogen peroxide [12]. The peroxide penetrates through the mitochondrial membrane and can also give rise to more hydroxyl and toxic radicals [13]. Hydroxyl radicals created in the mitochondria may damage mitochondrial proteins, fats and DNA. [14] There are several external factors that can increase oxidative stress and therefore increase the formation of ROS under stress conditions in plants, including atmospheric pollutants such as ozone and sulfur 
dioxide, Heavy metals, herbicides, as well as draft conditions. ROS is also produced in other organisms, including humans as part of healthy metabolism and as a result of several external factors such pollution, as smoke, exposure to industrial chemicals, ozone, ultraviolet radiation, $[15,16]$ Internal sources of ROS generation in humans include the mitochondria, peroxisomes, inflammation Ksntin oxidase and physical activity [10]. ROS in biological systems contributes to damage cellular proteins, DNA and lipids [17].

Compounds such as flavonoids and anthocyanins

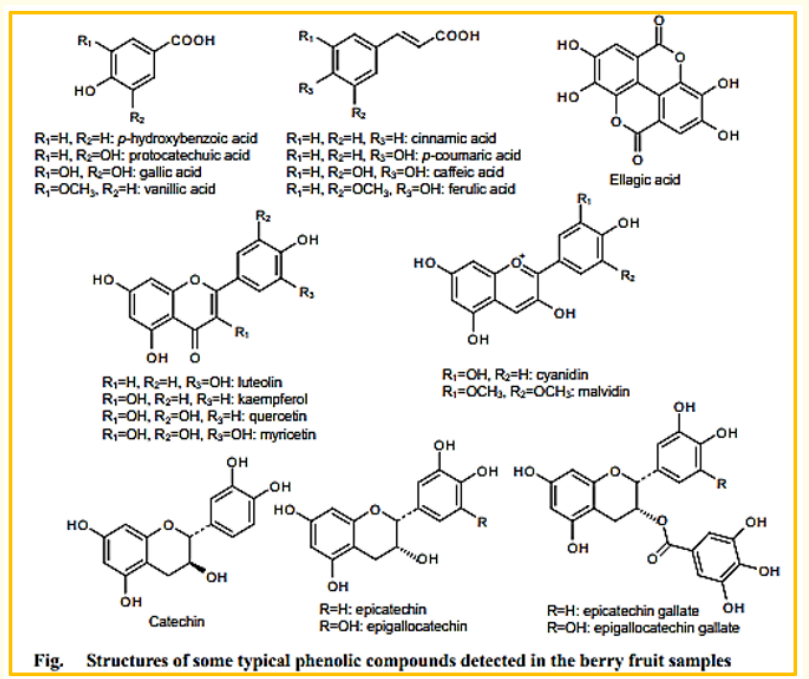

Figure 1

Fruits and vegetables contain various phytonutrients, many of which have antioxidant properties [18]. Studies confirmed that fruits and vegetables contain Other antioxidants, in addition Vitamins are know. Blueberries are a kind of berries which is also very tasty for our own health. In a study conducted by Patras University, their value for treating diseases such as Parkinson's disease (PD), which affects a large proportion of the elderly population is emphasized as C and E, or carotenoids, that Significantly contribute to their overall antioxidant capacity, Flavonoids (including compounds such as flavones, isoflavones, flavanones, anthocyanins, and ketones) Ingredients of fruits and vegetables have a potent antioxidant. There is compelling epidemiological evidence to show these Fruits and vegetables are usually beneficial to health And contribute to the prevention of near processes. Therefore, it is essential to Characterize the beneficial phytonutrients present These foods and the mechanisms responsible for its Effects.

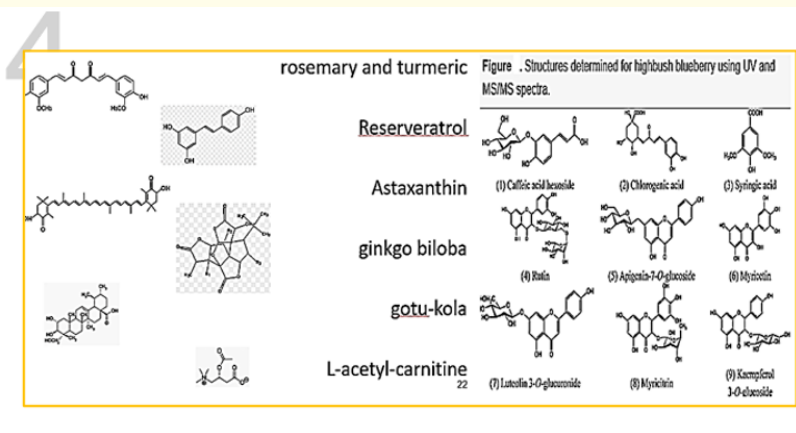

Structures of antioxidants that cross the BBB

Structures determined in blueberries

Figure 2

Why we can not live without antioxidants? Metabolic processes in the brain use antioxidants to prevent damages of oxidative stress. They prevent oxidative stress that leads to neurodegeneration $[19,20]$.

Plants are immobile and can not escape from negative environmental influences as easily as others Organisms such as animals. For this reason, plant systems have adapted to survive in extremes Conditions, such as lighting, high temperature, draft, and freezing, which can lead to Oxidative damage due to excessive production of free radicals. These have developed antioxidants Mechanisms involve a wide variety of compounds, including superoxide dismutase (SOD), catalase, and essential antioxidants such as ascorbate, glutathione, tocopherol, and carotenoids Phenolic Recent studies have tied the high antioxidant activity of blueberries with their overall phenolic content. Phenols include a group of compounds called stilbenes. This article discusses the study to determine the presence of reverences [21], pterostilbene, and piceatannol (Figure) in blueberries by gas chromatography-mass spectrometry (GC-MS) in selected ion monitoring mode

The most radical scavengers (NOX) are antioxidants and phenols. They convert peroxyl radicals into hydroperoxides and they themselves are converted into FOXY radicals. Phenoxy radical can in various ways respond with radical and other radical resulting in nonradical products (Scheme). However, Foxy radicals formed also are capable of abstracting hydrogen from a polymer chain, for example, and thus in this way start a new oxidation cycle. Most phenolic antioxidants contain two tertiary butyl groups on 2 and 6 positions; These groups are able to protect the generated phenoxy 
radicals (stray barrier) and prevent initiation of a new oxidation cycle. However, presenting a sterile barrier also causes a reduction in the radical scavenging rate. Reducing stray barrier results in increased activity in scavenger radicals, but created phenoxy radicals can initiate new oxidation cycles. The ratio between increased basic growth rate and initiation rate will determine the effectiveness of antioxidants [22].

Styrene-based polyphenols
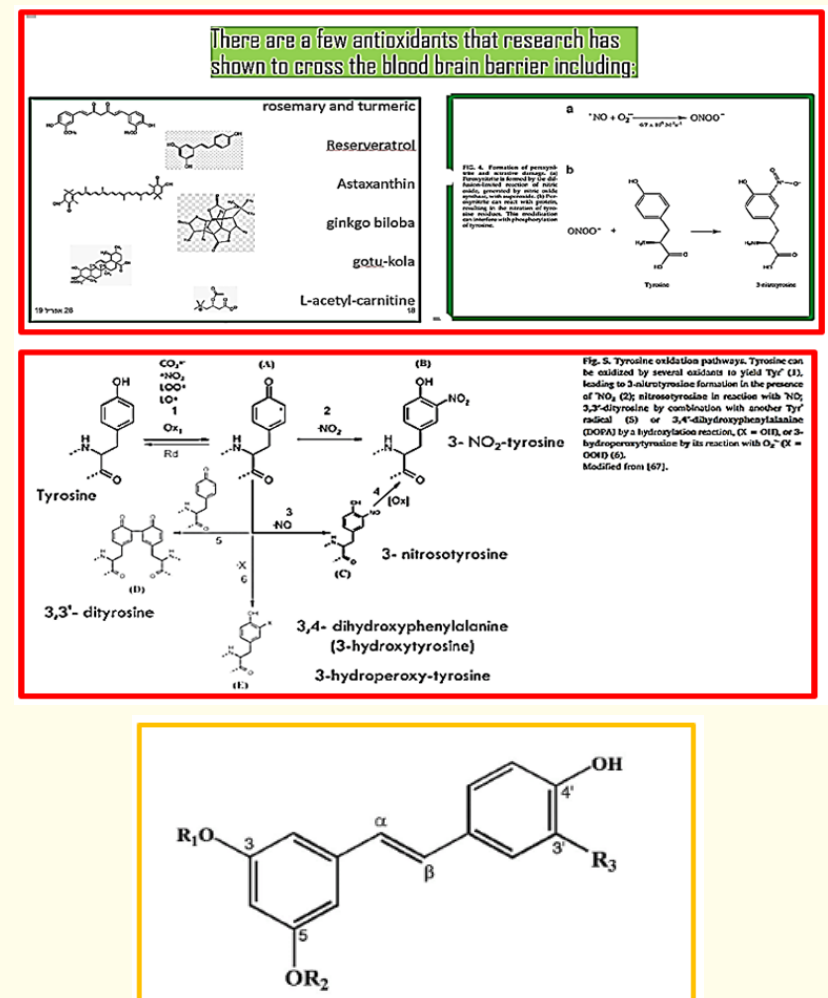

Resveratrol: $\mathrm{R}_{1}=\mathrm{R}_{2}=\mathrm{R}_{3}=\mathrm{H}$

Pterostilbene: $\mathrm{R}_{1}=\mathrm{R}_{2}=\mathrm{CH}_{3}, \mathrm{R}_{3}=\mathrm{H}$

Piceatannol: $\mathrm{R}_{1}=\mathrm{R}_{2}=\mathrm{H}, \mathrm{R}_{3}=\mathrm{OH}$

Figure 3: Structures of Resveratrol Pterostilbene And Piceatannol Reprinted With Permission From The Journal of Agricultural And Food Chemistry Copyright 2004 American Chemical Society.

SCHEME: Reactions of a phenolic antioxidant with peroxy radicals.

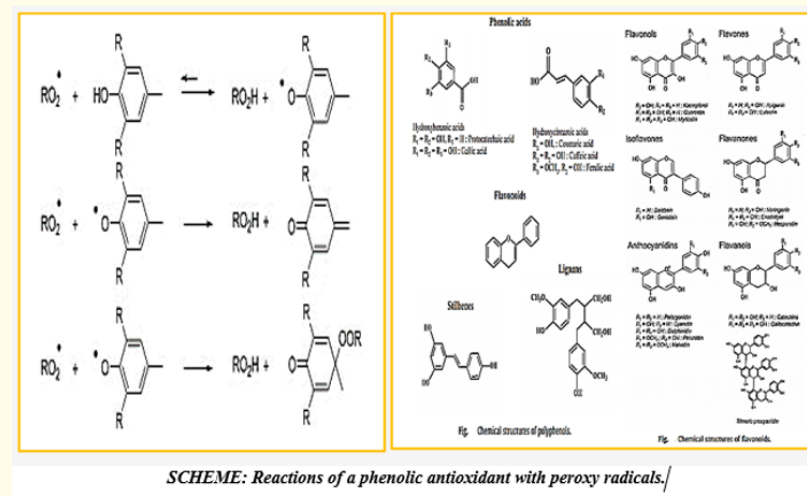

Figure 4

But what are the antioxidants

Antioxidants are naturally occurring chemicals that prevent aging and chronic diseases. The more we have our bodies equipped to fight infections, infection and cancer.

The blueberries contain Phenolic acids are non-flavonoid polyphenolic compounds Which can be distinguished as benzoic acid and cinnamic acid Derivatives based on the C1 - C6 and C3 - C6 spine, respectively [23].

\section{And where did they come from?}

Antioxidants are chemical agents are found naturally in many foods such as fruits, vegetables, tea, wine and chocolate. You may have heard that broccoli, blueberry bar, pomegranate, dark chocolate Goji berries are rich in antioxidants. Matcha Green Tea contains more antioxidants than any other food. If broccoli has 31 units of antioxidant per gram, blueberries bar 93 units per gram, at 253 goji berries per gram, Matcha Green Tea has 1384 units of antioxidant per gram! (Figure 5).

The effect of blueberry (PrB) extract rich in polyphenol from wild was studied after 7 days of intraperitoneal administration (an adult male b alb c mice: (a) on the cognitive function of the muscles and (b) in the antioxidant state and in the activity of acetylcholine-esterase (AChE), an enzyme Degrades acetylcholine (ACH), a neurotransmitter that plays an essential role in cognitive functions in whole brain homogenates. The conclusion of the study showed that individual components of blueberries, polyphenols, which Purple colour on fruits and vegetables where they are included, can impro- 
ve memory as well as the learning ability To protect the nervous function of the brain from the deterioration that appears over the years.

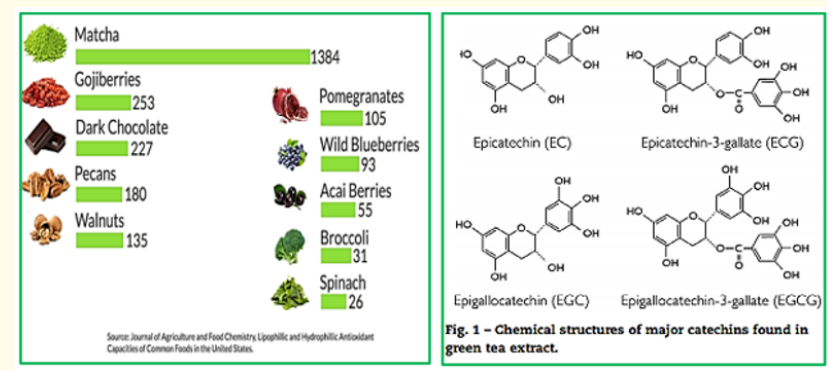

Figure 5

\section{What is MATCHA Green TE anyway?}

Matcha is a sort of green tea grown in the hills of Japan produced by tahina leaves and young tea into light green powder. Green Tea Matcha contains powerful antioxidants known as catechins which are not found in other foods. Catch chin's main matcha is EGCG (epigallocatechin gallate) known for its strong cancer properties.

Why are we a very psychiatrist on MATCHA green tea skin care?

The same antioxidants that make green tea and protective gout also support skin health by reducing inflammation and neutralizing free radicals, results of the oxidative stress, that accelerate aging. This is the very reason why there is an increasing number of skin care products claiming to contain Matcha.

Vanga Lama's Matcha Green Tea Face Oil brings the power of antioxidants to your fingertips to enjoy your skin.

Vanga lama's tea green tea is $\mathbf{1 0 0 \%}$ pure tea compatible

The above diagram points out the by far antioxidants abundance of the matcha tea. However, Effectively and weight wise, blueberries are consumed in foods and baking foods more than matcha tea. Therefore it might be useful to discuss its benefits to our health and welfare (Figure 6).

Antioxidant activity and the phenolic composition [24] of six blueberry seedlings.

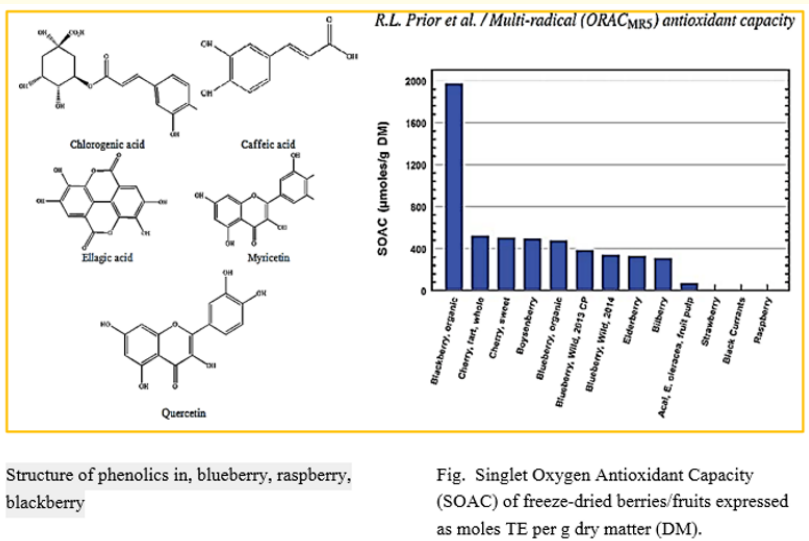

Figure 6

The microbial axis of the stomach brain has received an abundance of attention recently. It is thought that the gut microbiota can affect brain function and behaviour, but how this happens is still unknown. It has been found that bacteria can enter the brain via a blood-brain barrier, and / or using nerves that innervate the intestines. Here we show the presence of bacteria in the human brain and mouse under non-harmful or non-traumatic conditions. It is found in bacteria, identified by morphological criteria, in ultrastructural samples of human post-mortem brain. Serial section analysis for identification and quantification. All the cases contained bacteria in varying amounts. Bacteria were rod-shaped, containing capsules, nucleotides, ribosomes and cavities. The average diameter of the short axis was $0.496 \mathrm{~mm}$, many were segmented, with a long axis averaging about $1.78 \mathrm{~mm}$ between sections, others were not seen to be segmented and were approximate (Figure 7).

$0.866 \mathrm{~mm}$ in the long axis, the vast majority of the profiles had a thick capsule of about $100 \mathrm{~nm}$, and the density of the bacteria varied according to the area of the brain, with abundant bacteria of the neutrophil, the hippocampus and the prefrontal cortex, but their number was thinned in the striatum. Of the blood-blood barrier, dendrites and soma of glial cells, they were also plentiful nearby and within the myelinated axons. To address the possibility that the bacterium in human tissue was the result of an afterlife object, we examined the mouse brain immediately determined by death $(\mathrm{n}=$ 10) In similar cells, in order to eliminate the possibility that the presence of bacteria is caused by infection $(\mathrm{N}=4)$ was treated in 
the same manner, and we found no bacteria, and the observation that the location of the bacterium was precise and deep inside the samples was also contagious against infection. Interestingly, there were no structural signs of inflammation in each brain tested. It is clear how the entry microbes enter the brain, but their evidence in the axons and blood-brain barrier support previous hypotheses.

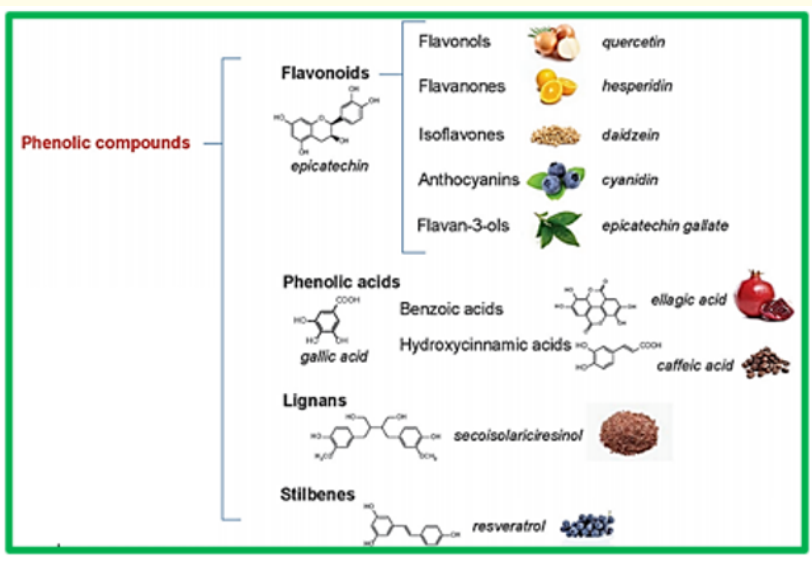

Figure 7: Phenolic compounds in "Mediterranean Diet" (credit ref. 8).

Alpha-synuclein $(\alpha S)$ is a neuronal cell protein associated with Parkinson's disease (PD). The accumulation of $\alpha \mathrm{S}$ within the ENS and its movement from the intestines to the brain are playing a vital role in the pathogenesis and progression of PD. AS has no known function in humans and the reason for its accumulation within the ENS is unknown. Several recent rodent studies have tied $\alpha \mathrm{S}$ immune activation of the central immune system. We assumed that $\alpha \mathrm{S}$ in ENS might play a role in the innate immune defence of the human digestive system (GI).

\section{Our brain is home to the microbial inhabitants}

Treatment of multiple neurodegenerative disorders will require the delivery of therapeutic protein to neurons or glial cells throughout the CNS. The presence of the blood barrier in the brain delays the supply of proteins from the blood, thus necessitating a new method of delivery.

Antimicrobial peptides (AMPs) are host-encoded antibiotics that fight invading microorganisms. These short, short peptides have been involved in many biological processes, particularly with innate immunity.
The penetration of microbes into the inner brain can be the onset of inflammation followed by infection in the brain. One can rationalize the digestion of APP and other proteins by enzymes to form antimicrobial peptides like The amyloid $b$ which combat the microbes, but aggregate to form many sorts of stable formations that damage the synapses as depicted above. The series of events that are induced by the oxidative stress of the inflammation, lead to the harming antimicrobial peptides of which the amyloids are only one sort. Since peptides can barely enter into the brain via the BBB [25]. However, it is possible to enter the brain via various receptors, an example is the diazepine receptor [26].

In innate immunity, immunity based on inflammation is the first line of protection against vertebrates against microorganisms. Inflammation relies on many cellular and molecular agents that can attack invasive pathogens very shortly after the interaction of inflammatory cells with the intruder, but not correctly. Because of this non-specific reaction, inflammation can produce significant costs for the host if the inflammatory response, and oxygen-related damage, out of control. This casts an intense selection pressure that acts to optimize the two main characteristics of the inflammatory response: the timing of activation and the solution (downregulation process of the reaction). Nevertheless, host immune regulation also opens the way to pathogens to subvert host defences.

Currently, many agree that the gut microbiome reaches the brain using the "gut-brain axis" and is the cause for the onset of neurodegeneration - this penetration results in inflammation, then infection and apoptosis of the brain tissue. Antimicrobial peptide surrogates [27], based on a brain-penetrating scaffold-like diazepine [28] or 1,4 dihydropyridine [29] may be suitable to combat the invading microbiota and in a later stage serve as antimicrobial agents to combat the infection and prevent aggregation. Blueberries aren't actually blue [30], but deep purple, which is the colour of anthocyanin, a The visible colour range of common anthocyanidins [31] (Figure 8).

The pigment is particularly rich in blueberries. Blueberries are known to be high antioxidants, "blueberries are the king of antioxidant food", which are suitable for the human body; Anthocyanin is considered to be useful in combating inflammation. The good rule is to follow, the darker the berry, the more anthocyanins exist. The primary antioxidant compounds in blueberries belong to a family of antioxidant polyphenols called flavonoids. The group of fla- 
vonoids in particular - anthocyanins — is thought to be responsible for much of these berries' beneficial health effects.

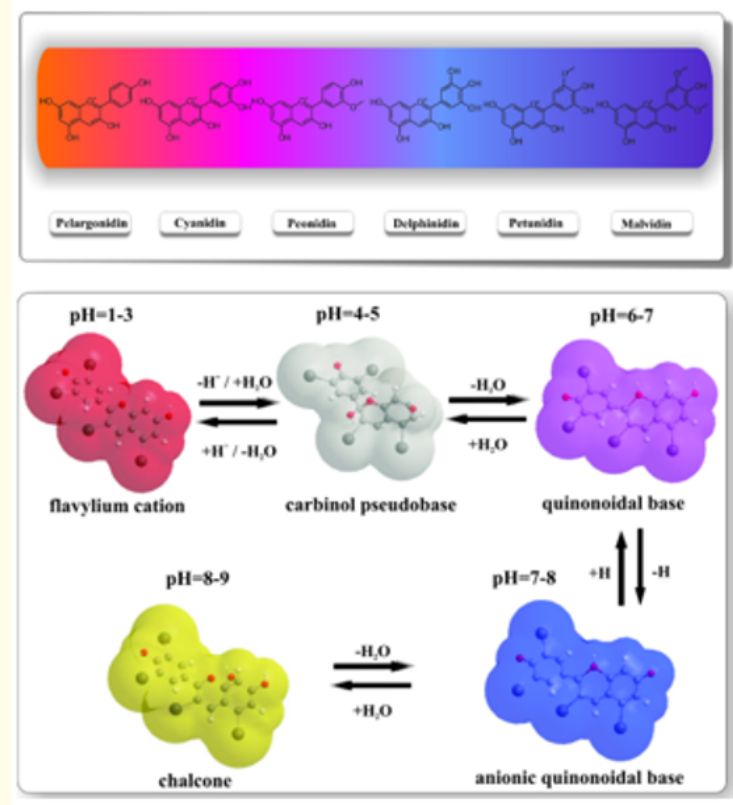

The visible color range of common anthocyanidins (credit ref. $\left.P^{2}\right]$ )

Figure 8

There are many antioxidants that may aid in this circumstance for an instant in a Mediterranean diet or by using forest berries, blueberries [32] that contain many sorts of Antioxidants. In fact, the consumption of blueberries significantly improves vascular function in healthy men in a time-dependent manner of intake. These benefits may be mechanically linked to the actions of cyclic phenolic metabolites on neutrophil activity in NADPH oxidase. The research yielded the identification of Phenolic Acids, Flavanol Glycosides and Antioxidant Potential in Blueberry, Blackberry, Raspberries and Cranberries were reported [33].

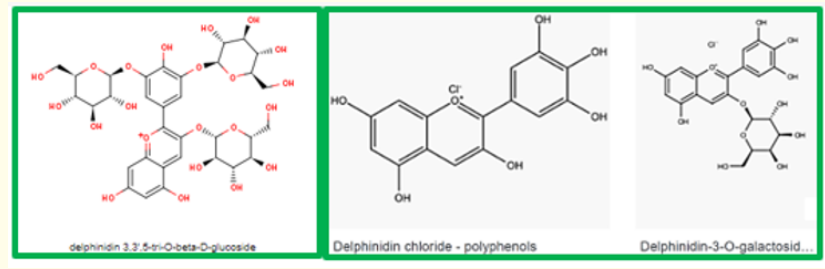

Figure 9
The daily nourishment of fruits and vegetables is consistently associated with reductions in the incidence of degenerative diseases, by having antioxidant (The blue pigment Delphinidin for example. Delphinidin is the most common anthocyanidin molecule in blue flowers and forms with pelargonidin and cyanidin the three anthocyanidins known as the principal and basic skeletons of flower colour pigments. so they are the most widespread in nature), anti-inflammatory and anticarcinogenic activity [34]. Scientists perceived [35], that daily intake of 600 grams of nutritious fruits or vegetables can prevent the risk of coronary heart disease by more than $31 \%$ and ischemic stroke by $19 \%$. Several epidemiological studies summarized in diverse papers [36-39] have built the consensus that diets rich in fruits and vegetables have beneficial effects on human health.

Phenolic acids and anthocyanidins in red fruits

Berries are characterized by high quantities of antioxidants molecules. These chemicals are a group of secondary metabolites that prevent the fruit due to oxidation, due to I factors such as light, air, oxygen and microbiological attacks. Antioxidants and phenols interfere with the oxidation process like free radicals terminals and sometimes also metal complex ants. Phenolic compounds or polyphenols are a group of highly sensitive hydroxylate molecules. Several studies have found that they have different biological properties, such as anti-probiotics, anti-diabetic, anti-cancer, anti-microbial, anti-inflammatory, anti-viral, and especially crucial for this criticism: antioxidants. They have different structures but generally contain an aromatic ring with one or more hydroxyl group. The radical drainage capacity of phenolic antioxidant molecules is based on the ability to become more stable radicals than most free radical species due to the stabilization of the free electron by the delocalization of the aromatic ring of the phenolic compounds. Classification of phenolic antioxidants can be made, the most important being phenolic acids and anthocyanins, a subgroup of flavonoids. Phenolic acids can be divided into two categories: hydroxybenzoic derivatives and hydroxyzine derivatives. The first group contains molecules such as hydroxybenzoic, corrugated, vanilla, and alginic acid (Figure. In the second group p-coumaric, caffeic, ferulic, chlorogenic and hydroxycinnamic acids can be found. These compounds can be found in extensive berries, and each type of berry contains a typical profile of phenolic molecules. Anthocyanins are water-soluble plant pigments responsible for blue, purple, and red dye of many plant tissues [40]. Anthocyanidins are based on flavylium ion, or a 2-phenylchromenylium variety of chemical groups 
that can replace the different positions (R1, R2...) to create the anthocyanins found in nature. Simplification of this ion, focusing on common structures in red fruits can be seen in the Figures below.

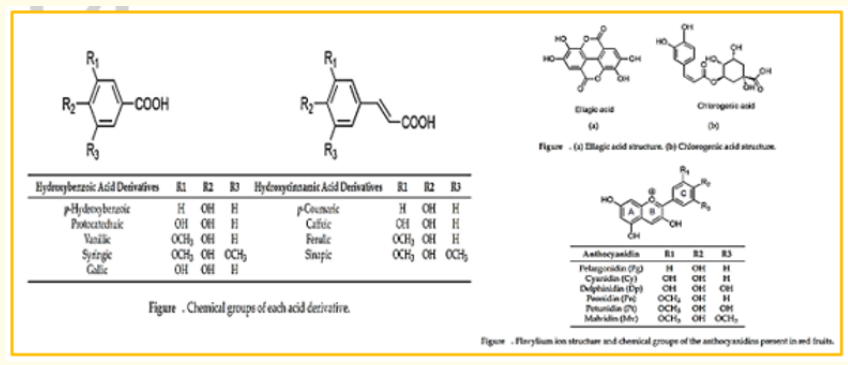

Figure 10

Flavylium ion structure and chemical groups of anthocyanins present in red fruits.

There are about 17 anthocyanins in nature, while only 6 of them, pelargonidin, cyanidin, delphinidin, petunidin, peonidin, and malvidin are present in most foods [41]. Anthocyanins are formed, when anthocyanidins are coupled to sugars. In red fruits, anthocyanins are mainly 3-glucosides of anthocyanins, cyanidin-3-glucoside being the most common compound of berries (among flavonoids, anthocyanins are antioxidants which play an essential role in reducing the risks of various degenerative diseases of the human [42] The stability of anthocyanidins depends on $\mathrm{pH}$. In acidic or alkaline $\mathrm{pH}$, the most phenolic groups of the protonate of anthocyanidins and detractors cause changes in the electronic distribution, which simultaneously affect the wavelength of perceived colour absorption.

\section{The pterostilbene antioxidants}

Pterostilbene [43] is found in almonds, various dust grains (including blueberries) vine leaves and vines, and Pterocarpus marsupium heartwood. grape leaves and vines, and Pterocarpus marsupium heartwood.

Pterostilbene (trance 3,5-dimethoxy-4-trihydroxystilbene) is a natural dietary compound and a major antioxidant component of blueberries. It increased bioavailability compared to other STYLBAN compounds, which may increase its nutritional benefits and possibly contribute to a valuable clinical effect. Many studies have demonstrated the antioxidant activity of pterostilbene both in vi- tro and in vivo models demonstrating both motivational and therapeutic benefits. The antioxidant activity of pterostilbene was involved in anti-carcinogenesis, modulation of neurological disease, anti-inflammatory, attenuation of vascular disease, and improvement of diabetes. In this review, we investigate the antioxidant properties of pterostilbene and its association with common pathways and pathways to give a summary of the clinical potential of pterostilbene in prevention and treatment in various medical conditions.

The structure of pterostilbene is very similar to resveratrol [44], Which is Inhibiting Islet Amyloid Polypeptide fibril Formation by the Red Wine Compound, although a 3.5-dihydroxy portion of resveratrol (two hydroxyl groups on the left benzene ring) are replaced with methoxy groups. The antioxidant capacities of resveratrol require that there is hydroxylation in position 4 (on the right side of the molecule) and that the general molecule is in a transrather than cis configuration. Stilbenes above all comply with these requirements while others such as pyroxylin not. Methoxy groups on pterostilbene appear to allow a relatively sizeable antioxidant capacity to resveratrol. Pterostilbene is methoxylated resveratrol, in fact. It has a more significant difference in structure than resveratrol than piceatannol (another joint stilbene compound, which is more similar to resveratrol). These three structures form common nutritional stilbenes $[45,46]$.

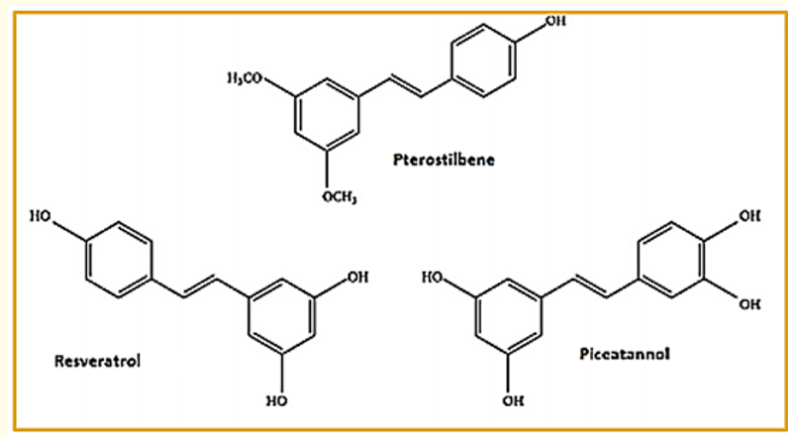

Figure 11

Pterostilbene is included in low quantities in a number of plants, especially blueberries and grapes. Despite being low concentration, it is reasonable to assume that bio-active following the oral ingestion of these fruits and their products (such as wine). 


\section{Longevity and life extension}

Pterostilbene mechanisms have been observed to upregulate a variety of mitochondrial genes in the yeast assay involved in respiratory, electron transport, mitochondrial targeting protein, and mitochondrial protein synthesis. The oxidative stress observed during the aging process in neurons appears to be weakened with low diet levels of pterostilbene (0.004- $0.016 \%$ of the diet), and enhanced with greater strength than resveratrol.

Pterostilbene (trans-3,5-dimethoxy-4'-hydroxystilbene), naturally an analogy dimethyl ether of resveratrol, was first isolated in Pterocarpus Santa Linus and is also found in several Types of berries and grapes. [47] in vitro and in vivo studies suggest that pterostilbene and its analogues have a number of biological activities Linked to AD, including antioxidants, anti-inflammatory drugs, and neurostimulation. Recently, many researchers have proven That pterostilbene analogues can inhibit self-aggregation induced $\mathrm{AB}$, Worsen the ab-induced poisoning, promote ab-approval and reduce [48]. Some studies have shown that it has the ability To inhibit monoamine oxidase (MAO) [49]. It can be concluded that pterostilbene may be used as a starting compound in the design of multiple-purpose drugs to treat AD. Our research group Was involved over the years in development Multi-purpose target as potential drug candidates for AD. B In particular, our group reported the synthesis of pterostilbene carbamate derivatives as cholinesterase inhibitors and double neuroprotective agents.14 Benzylamines have been shown to be essential cholinesterase inhibits our previous pharmacophore work [50] as further development of our work on design Synthesis of new multifunctional compounds with better pharmacological profiles, here we aim to use multi-purpose directed Drug design strategy combine pterostilbene with benzylamines Using a chain of amide of different lengths [51].

The multiple benefits of pterostilbene in the treatment and prevention of human diseases have been attributed to the anti-oxidant, anti-inflammatory, and anticycinogenic properties that result in improved function of normal cells and inhibitions of malignant cells $[52,53]$. Treatments with blueberry extract in PM and similar disease models have yielded parallel results possibly due to antioxidant activity underlying mechanisms of pterostilbene. The evidence presented in this review shows that pterostilbene reduces oxidative stress (OS) and production of reactive oxygen species (ROS), such as hydrogen peroxide $\left(\mathrm{H}_{2} \mathrm{O}_{2}\right)$ and anion sulfoxide, whi- ch are involved in initiating pathogenesis of some disease processes [54]. In addition, different cell lines treated with pterostilbene showed increased expression of antioxidant catalase, total glutathione (GSH), glutathione peroxidase (GPx), glutathione reductase (GR), and superoxide dismutase (SOD). In this review, the clinical potential and antioxidant potential of pterostilbene in a number of disease systems will be reviewed and summarized.

\section{Concluding remark}

Pterostilbene's are nutritional antioxidants (pronounced "Tero-STILL-Bean") yet. But this is the prospect of compound berry inhibitor. Breast cancer, diabetes, LDL cholesterol in humans may soon make it known as other natural health enhancement materials.

The announcements aroused a wave of attention to Pterostilbene [55], not only in the United States but also in other countries. At least two UK news organizations have directly attributed a boom in British blueberry sales to Rimando's findings. And Oxford's laboratory results have since been cited by companies marketing products ranging from blueberry extract to concentrate juice to commercially available pterostilbene itself.

Combining the results of past and present experiments Different disciplines, biochemistry, cell biology, physiology, Pathophysiology, epidemiology and food chemistry, It will be necessary to identify the most effective polyphenols to determine the optimal levels of intake for better health. God The current research efforts will be coordinated with current efforts Identify more accurate biomarkers of dietary-related risks Diseases should lead to nutritional recommendations and The formulation of new food products that contribute to good health.

\section{Bibliography}

1. Augustin Scalbert., et al. "Polyphenols: antioxidants and beyond". American Society for Clinical Nutrition 81 (2005): 215S217S.

2. Wu-yang Huang., et al. "Survey of antioxidant capacity and phenolic composition of blueberry, blackberry, and strawberry in Nanjing". Journal of Zhejiang University Science B 13.2 (2012): 94-102.

3. James A Joseph., et al. "Reversals of Age-Related Declines in Neuronal Signal Transduction, Cognitive, and Motor Behavioral Deficits with Blueberry, Spinach, or Strawberry Dietary Supplementation". The Journal of Neuroscience 19.18 (1999): 8114-8121. 
4. T Tamada. "Determination of Stilbenes in Blueberries". Food Style 21.6 (2002): 55-59.

5. Kuresh A Youdim., et al. "Short-Term Dietary Supplementation of Blueberry Polyphenolics: Beneficial Effects on Aging Brain Performance and Peripheral Tissue Function". Nutritional Neuroscience 3 (2000): 383-397.

6. Andrea Cignarell., et al. "Novel Lipid-Lowering Properties of Vaccinium Myrtillus L. Leaves, A Traditional Antidiabetic Treatment, In Several Models of Rat Dyslipidaemia: A Comparison with Ciprofibrate". Thrombosis Research 84 (1996): 311-322.

7. Halliwell B. "Oxidative Stress and Neurodegeneration: Where are we Now?" Journal of Neurochemistry 97 (2006): 16341658.

8. Perl-Treves R and Perl A. “Oxidative stress: An introduction. In Oxidative Stress in Plants; Inze, D., Montago, M., Eds.; Taylor and Francis Publishers: New York, NY, USA (2002): 1-2.

9. Yu BP. “Cellular Defenses against Damage from Reactive Oxygen Species". Physiological Reviews 74 (1994): 139-162.

10. Rhoads DM., et al. "Mitochondrial Reactive Oxygen Species. Contribution to Oxidative Stress and Interorganellar Signaling". Plant Physiology 141 (2006): 357-366.

11. Puntarulo S., et al. "Hydrogen Peroxide Metabolism in Soybean Embryonic Axes at the Onset of Germination". Plant Physiology 86 (1988): 626-630.

12. Bowler C., et al. "Superoxide Dismutase and Stress Tolerance". Annual Review of Plant Biology 43 (1992): 83-116.

13. Moller IM. "Plant Mitochondria and Oxidative Stress: Electron Transport, NADPH Turnover, and Metabolism of Reactive Oxygen Species". Annual Review of Plant Biology 52 (2001): 561591.

14. Kasai H., et al. "Formation of 8-Hydroxyguanine Moiety in Cellular DNA by Agents Producing Oxygen Radicals and Evidence for its Repair". Carcinogenesis 7 (1986): 1849-1851.

15. Pell EJ., et al. "Ozone-Induced Oxidative Stress: Mechanisms of Action and Reaction". Physiologia Plantarum 100 (1997): 264-273.

16. Heck WW. "Factors Influencing Expression of Oxidant Damage to Plants". Annual Review of Phytopathology 6 (1968): 165-188.
17. Slemmer JE., et al. "Antioxidants and Free Radical Scavengers for the Treatment of Stroke, Traumatic Brain Injury and Aging". Current Medicinal Chemistry 15 (2008): 404-414.

18. Ronald L Prior., et al. "Antioxidant Capacity as Influenced by Total Phenolic and Anthocyanin Content, Maturity, and Variety of Vaccinium Species". Journal of Agricultural and Food Chemistry 46 (1998): 2686-2693.

19. David Vauzour. "Dietary Polyphenols as Modulators of Brain Functions: Biological Actions and Molecular Mechanisms Underpinning Their Beneficial Effects". Hindawi Publishing Corporation Oxidative Medicine and Cellular Longevity (2012): 914273.

20. Genevieve Bureau., et al. "Resveratrol and quercetin, two natural polyphenols, reduce apoptotic neuronal cell death induced by neuroinflammation". Journal of Neuroscience Research 86 (2008): 403-410.

21. Erin Kelly., et al. "Biochemical Properties and Neuroprotective Effects of Compounds in Various Species of Berries". Molecules 23.1 (2018): 26.

22. Elmer R Altwicker. "The Chemistry of Stable Phenoxy Radicals". Chemical Reviews 67.5 (1967): 475-531.

23. Alfredo Cassano., et al. "Effect of polyphenols-membrane interactions on the performance of membrane-based processes. A review". Coordination Chemistry Reviews 351 (2017): 45-75.

24. Rodrigo A Contreras., et al. "In Vitro Cultivars of Vaccinium corymbosum L. (Ericaceae) are a Source of Antioxidant Phenolics". Antioxidants 4.2 (2015): 281-292.

25. William A Banks. "Peptides and the blood-brain barrier". Peptides 72 (2015): 16-19.

26. Moshe Gavish., et al. "Enigma of the Peripheral Benzodiazepine Receptor". Pharmacological Reviews 51.4 (1999): 629-650.

27. Shimon Shatzmiller., et al. "Combatting the Microbial Onset of Neurodegeneration the Peptide Surrogate Approach". EC Pharmacology and Toxicology 6.3 (2018): 152-184.

28. Galina M Zats., et al. "Antimicrobial benzodiazepine-based short cationic peptidomimetics". Journal of Peptide Science 21 (2015): 512-519.

29. Inbal Lapidot., et al. "1,4-Dihydropyridine Cationic Peptidomimetics with Antibacterial Activity". International Journal of Peptide Research and Therapeutics 21 (2015): 243. 
30. https://www.theguardian.com/lifeandstyle/2014/jul/12/ ask-a-grown-up-why-are-blueberries-blue

31. Anthony Ananga., et al. "Production of Anthocyanins in Grape Cell Cultures: A Potential Source of Raw Material for Pharmaceutical, Food, and Cosmetic Industries". DOI (2013): $10.5772 / 54592$.

32. Ana Rodriguez-Mateos., et al. "Intake and time dependence of blueberry flavonoid-induced improvements in vascular function: a randomized, controlled, double-blind, crossover intervention study with mechanistic insights into biological activity". The American Journal of Clinical Nutrition 98.5 (2013): 11791191.

33. Zorița Diaconeasa., et al. "Hplc/Pda-Esi/Ms Identification of Phenolic Acids, Flavonol Glycosides and Antioxidant Potential in Blueberry, Blackberry, Raspberries and Cranberries". Journal of Food and Nutrition Research 2.11 (2014): 781-785.

34. Yun JM., et al. "Delphinidin, an anthocyanidin in pigmented fruits and vegetables, induces apoptosis and cell cycle arrest in human colon cancer HCT116 cells". Molecular Carcinogenesis 48.3 (2009): 260-270.

35. Lock K., et al. "The global burden of disease attributable to low consumption of fruit and vegetables: implications for the global strategy on diet". Bulletin of the World Health Organization Journal 83.2 (2005): 100-108.

36. Laura Este'vez and Ricardo A Mosquera. "Molecular Structure and Antioxidant Properties of Delphinidin". The Journal of Physical Chemistry A 112 (2008): 10614-10623.

37. Hertog MGL., et al. "Dietary antioxidant flavonoids and risk of coronary heart disease: the Zutphen Elderly Study". Lancet 342 (1993): 1007-1011.

38. Trichopoulou A and Vasilopoulou. "Mediterranean diet and longevity”. British Journal of Nutrition 84 (2000): 205-209.

39. Silvina Bartesaghi and Rafael Radi. "Fundamentals on the biochemistry of peroxynitrite and protein tyrosine Nitration". Redox Biology 14 (2018): 618-625.

40. Galván D’Alessandro L., et al. "Kinetics of ultrasound assisted extraction of anthocyanins from Aronia melanocarpa (black chokeberry) wastes". Chemical Engineering Research and Design 92 (2014): 1818-1826.
41. Wu X., et al. "Concentrations of anthocyanins in common foods in the United States and estimation of normal consumption". Journal of Agricultural and Food Chemistry 54 (2006): 40694075 .

42. Paes J., et al. "Extraction of phenolic compounds and anthocyanins from blueberry (Vaccinium myrtillus L.) residues using supercritical CO2 and pressurized liquids". The Journal of Supercritical Fluids 95 (2014): 8-16.

43. Denise McCormack and David McFadden. "A Review of Pterostilbene Antioxidant Activity and Disease Modi_cation". Oxidative Medicine and Cellular Longevity (2013): 575482.

44. Rajesh Mishra., et al. "Inhibiting Islet Amyloid Polypeptide Fibril Formation by the Red Wine Compound Resveratrol". European Journal of Chemical Biology 10 (2009): 445-449.

45. Z Horváthová-Kozics K. "Structure-activity relationship of trans-resveratrol and its analogues". Neoplasma 52.6 (2005): 450-455.

46. Hasiah AH., et al. "Cytotoxic and antioxidant effects of methoxylated stilbene analogues on HepG2 hepatoma and Chang liver cells: Implications for structure activity relationship". Human and Experimental Toxicology 30.2 (2011): 138-144.

47. Agnes M Rimando., et al. "Cancer Chemopreventive and Antioxidant Activities of Pterostilbene, a Naturally Occurring Analogue of Resveratrol". Journal of Agricultural and Food Chemistry 50.12 (2002): 3453-3457.

48. Riviere C., et al. "Inhibitory activity of stilbenes on Alzheimer's $\beta$-amyloid fibrils in vitro". Bioorganic and Medicinal Chemistry 15 (2007):1161.

49. Pan LF., et al. "Design, synthesis and evaluation of novel tacrine-( $\beta$-carboline) hybrids as multifunctional agents for the treatment of Alzheimer's disease". Bioorganic and medicinal chemistry 22.21 (2014): 6089-6104.

50. Sang ZP., et al. "Design, synthesis and evaluation of novel tacrine-( $\beta$-carboline) hybrids as multifunctional agents for the treatment of Alzheimer's disease". European Journal of Medicinal Chemistry 94 (2015): 350.

51. YuxingLi., et al. "Pterostilbene-0-acetamidoalkylbenzylamines derivatives as novel dual inhibitors of cholinesterase with anti- $\beta$-amyloid aggregation and antioxidant properties for the treatment of Alzheimer's disease". Bioorganic and Medicinal Chemistry Letters 26.8 (2016): 2035-2039. 
52. AM Rimando., et al. "Cancer chemopreventive and antioxidant activities of pterostilbene, a naturally occurring analogue of resveratrol". Journal of Agricultural and Food Chemistry 50.12 (2002): 3453-3457.

53. AM Remsberg., et al. "Pharmacometrics of pterostilbene: preclinical pharmacokinetics and metabolism, anticancer, antiin?ammatory, antioxidant and analgesic activity". Phytotherapy Research 22.2 (2008): 169-179.

54. AAM Adly. "Oxidative stress and disease: an updated review". Research Journal of Immunology 3.2 (2010): 129-145.

55. Pterostilbene's "Healthy Potential Berry compound may inhibit breast cancer and heart disease". Agricultural Research (2006).

Volume 2 Issue 12 December 2019

C) All rights are reserved by Shimon Shatzmiller. 